\title{
Integrating Biodegradable 3-Dimensional-Printing into Tracheal Reconstruction
}

\author{
Todd A. Goldstein 1,2, Lee P. Smith ${ }^{2,3}$, Benjamin D. Smith ${ }^{1,2}$, \\ Daniel A. Grande ${ }^{1,2,4}$, David Zeltsman ${ }^{2,5}$ \\ ${ }^{1}$ Orthopedic Research Laboratory, Feinstein Institute of Medical Research, Manhasset, USA \\ ${ }^{2}$ Hofstra North Shore-LIJ School of Medicine, Hempstead, USA \\ ${ }^{3}$ Division of Pediatric Otolaryngology, Steven and Alexandra Cohen Children's Medical Center, New Hyde Park, \\ USA \\ ${ }^{4}$ Department of Orthopedic Surgery, North Shore-LIJ, Manhasset, USA \\ ${ }^{5}$ Department of Cardiovascular and Thoracic Surgery, Long Island Jewish Medical Center, New Hyde Park, \\ USA \\ Email: tgoldstein@nshs.edu
}

Received 10 August 2015; accepted 12 September 2015; published 15 September 2015

Copyright (C) 2015 by authors and Scientific Research Publishing Inc.

This work is licensed under the Creative Commons Attribution International License (CC BY). http://creativecommons.org/licenses/by/4.0/

(c) (i) Open Access

\begin{abstract}
Background: Currently, there is no set standard treatment for long-segment tracheomalacia and stenosis. In this study we set out to explore the potential to create a tissue engineered, biodegradable and three-dimensionally (3D) printed tracheal ring as a first step towards bioengineering a long segment tracheal replacement. Method of Approach: A 3D-Computer aided design (CAD) model was produced with multiple channels to allow for cellular growth while mimicking the native anatomy. The design was optimized to allow for printability, cellular expansion, and integration and 3D printed using a modified commercial 3D printer. Results: The cells grown in the scaffold demonstrated a similar proliferation trend compared to control. Chondrocytes within the 3D printed ring retained their phenotypic properties and did not infer any significant change in flexibility, contour and strength to the scaffold. Conclusion: The combination of living cells and a 3D modeled patient specific graft may address some of the unmet clinical needs in the field of tracheal reconstruction. This proof of concept study represents a first step towards producing a 3D printed and tissue engineered long segment tracheal replacement graft for airway surgery.
\end{abstract}

\section{Keywords}

Trachea, Tissue Engineering, 3D Printing, Three Dimensional Printing, Bioengineering 


\section{Introduction}

Stenosis or malacia of the trachea can result in difficult breathing, exercise intolerance, sleep apnea, tracheotomy dependence and/or death [1]. When the malacia or stenosis is restricted to the upper trachea or subglottis, surgeons can bypass the narrowing with a tracheotomy. In certain situations, where a short segment of the cartilage rings is either destroyed, patients may be candidates for either surgery to remove the extrinsic compression or tracheal resection [2]. In non-neoplastic cases, intraluminal silicone stenting can provide relief, albeit with the very significant risks of mucous plugging, infection or migration [3]. In neoplastic cases, the affected section must be removed.

Up to $30 \%$ of the tracheal length can be excised and the two ends can be sutured together with a tension-free anastomosis. If more than $30 \%$ of the trachea is excised, released maneuvers are often required to allow for a tension free closure. To address long-segment stenosis ( $>50 \%$ of the trachea) or revision tracheal resection where an adequate length of trachea is not available, a variety of tracheoplasty techniques have been attempted with varying degrees of success. There is clearly an upper limit to the length of trachea that is possible to safely resect and, thus, a segment of patients for whom an adequate surgical solution is not readily available. In order to address the upper limit of length, one can turn to create a biologic implant utilizing the patient's own cells, potentially placing the harvested cells within a scaffold. However, harvesting tracheal chondrocytes from human patients is likely not feasible. There are many other less invasive sources of chondrocytes available for use, including nasal and auricular that have been shown to produce cartilage in tissue engineering applications [4] [5]. Additionally, the use of marrow stromal cells in cartilage tissue engineering is a promising development for future clinical translation [6] [7].

Scaffold-based tracheal reconstruction has been investigated in pre-clinical models. However, few have been translated to clinical applications. A 3D printed external splint made of polycaprolactone has been investigated in 3 pediatric patients to treat tracheobronchomalacia, but will not suffice as a treatment for neoplasia [7]. A bioengineered and trachealbronchial replacement was implanted in a human patient with primary tracheal cancer for whom other reconstructive options were not possible. This graft was fabricated from polyhedral oligomeric silsesquioxane (POSS) covalently bonded to poly-[carbonate-urea] urethane (PCU) (POSS-PCU), and seeded with autologous mononuclear cells. However, the fabrication process and pre-implantation culture technique are exceedingly complex, prohibiting widespread use and may not be necessary [7] [8].

This report details a method of creating a three-dimensionally (3D) printed and tissue engineered biodegradable tracheal ring that is seeded with chondrocytes as a first step towards addressing this unmet clinical need.

\section{Material and Methods}

\subsection{Designing, Printing, and Culturing of Scaffold}

A New Zealand white rabbit's tracheal ring was cross-sectionally dissected and scanned to serve as a template for the model. A 3D-Computer aided design (CAD) model was created using the Rhino3d ${ }^{\mathrm{TM}}$ Wenatchee-OsX CAD designer. The design was modeled to mimic native anatomy while allowing for printability, cellular expansion, and integration (Figure 1). The scaffold had an inner lumen of $6 \mathrm{~mm}$, an outer diameter of $8 \mathrm{~mm}$ and a height of $2 \mathrm{~mm}$. The ring was $1 \mathrm{~mm}$ thick with porosity ranging from 200 to 600 microns.
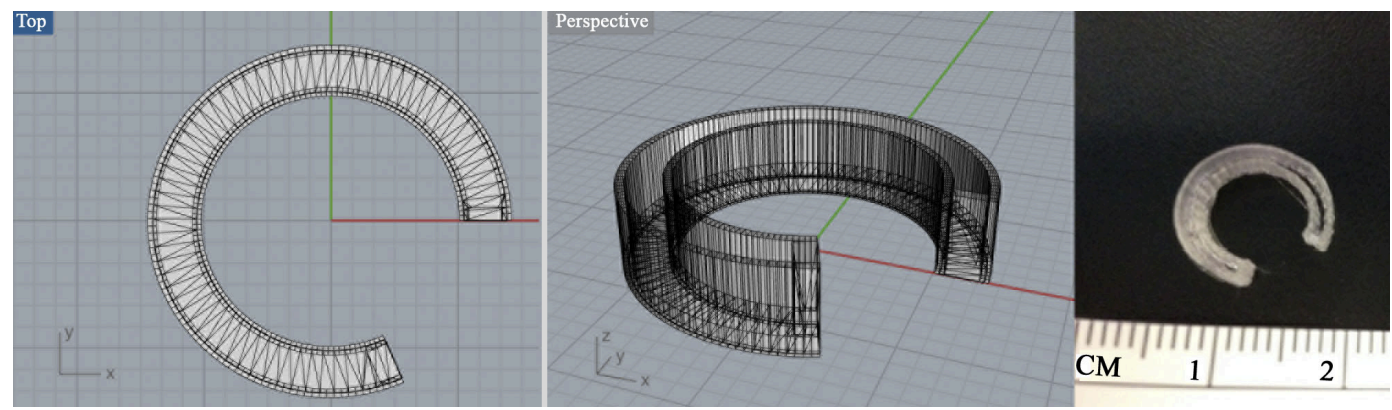

Figure 1. Top and perspective views of the CAD design (tracheal ring). Multiple channels within the wire diagram shown allow foe cellular incubation and growth. Gross photo contains grid like pattern seen in Figure 3 and Figure 4 in the base (1 mm box scale). 
The design template was optimized for 3D printing in MakerWare software (MakerBot ${ }^{\circledR}$, Brooklyn NY).

Scaffolds were 3D printed on a Makerbot Replicator Desktop Printer, using poly-lactic acid (PLA, Makerbot, Brooklyn NY, mw-128 kDa). Cells were suspended in $4 \mathrm{ml}$ type-1 collagen gel, as described in the Gel Preparation section. Approximately $50 \mu \mathrm{L}$ (300,000 cells) of the suspension was injected into each scaffold with a 22 gauge flat needle. The completed scaffold was removed from the build plate, and placed in culture media (DMEM/F-12 50/50 1x media (Cellgro, Manassas, VA) with 10\% FBS and a 1\% antibiotic-antimycotic solution (Cellgro, Manassas, VA)). Samples were incubated at $37^{\circ} \mathrm{C}, 5 \% \mathrm{CO}_{2}$, and $95 \%$ humidity for the duration of their time points. 32 scaffolds were divided into three groups: 1-empty scaffold, 2-collagen control, and 3-cell seeded scaffold.

\subsection{Isolation and Culture of Cells}

Chondrocytes were harvested from 4 New Zealand white (NZW) rabbit tracheal rings. NZW's were obtained from control samples immediately post-mortem of an alternate study, not involving the trachea, with the institutional animal care and use committee's approval. The cartilage samples were placed a collagenase solution (2.4 mg of collagenase per $\mathrm{ml}$ of DMEM) and digested at $37^{\circ} \mathrm{C}$ for 4 hours in a spinner flask. After digestion, the cell suspension was sieved through a $100 \mu \mathrm{m}$ cell strainer twice. Undigested pieces of tissue were discarded. The cell suspension was centrifuged at $1500 \mathrm{rpm}$ for 10 minutes, counted and plated in culture media at a density of $10,000 \mathrm{cells} / \mathrm{cm}^{2}$. Cells were expanded out three passages and then used for the preparation of the gel bioink. Prior to use, a sample of the isolated chondrocytes were visualized and stained with hematoxylin \& eosin and Safranin O/Fast Green to confirm chondrocytic lineage.

\subsection{Gel Preparation}

Collagen type 1 gel was produced by combining $8 \mathrm{~mL}$ of PureCol Purified Bovine Collagen Solution (3.1 $\mathrm{mg} / \mathrm{mL}$ ) (Advanced BioMatrix, San Diego, CA) with $1 \mathrm{~mL}$ of 10X RPMI (Sigma-Aldrich, St. Louis, MO). 1M $\mathrm{NaOH}$ was added dropwise until the solution turned uniformly pink on ice indicating a neutral $\mathrm{pH}$. The final collagen concentration was $2.7 \mathrm{mg} / \mathrm{mL}$. The gel was stored at $4^{\circ} \mathrm{C}$ until used to resuspend cell pellets when needed.

\subsection{Cell Proliferation Assay}

3D printed cell seeded scaffolds (group 3) were analyzed for cell proliferation after 1, 3, 5 and 7 days using the MTS assay (Promega, Madison, WI). At each time point, scaffolds were removed from their cell culture media, rinsed with HBSS (Corningware, Corning, NY), and incubated in $500 \mu \mathrm{L}$ of culture media with $125 \mu \mathrm{L}$ MTS for 90 minutes. Three $100 \mu \mathrm{L}$ aliquots of each sample's incubation medium were transferred to a 96-well plate and their absorbance measured at $492 \mathrm{~nm}$ using an ELX800 plate reader (Biotek, Winooski, VT).

\subsection{Cell Labeling}

Chondrocytes were labeled prior to printing to visualize distribution throughout the scaffold. Chondrocytes were centrifuged at $250 \mathrm{~g}$ for 5 minutes; the supernatant was carefully discarded and replaced with cell labeling solution, $50 \mu \mathrm{L}$ of fresh PBS and $50 \mu \mathrm{L}$ of $2 \mu \mathrm{M}$ Calcein AM per sample. Cells were re-suspended in the working solution and incubated for 30 minutes. Cells were centrifuged again for 5 minutes at $250 \mathrm{~g}$ and the supernatant was removed. Cells were re-suspended in type- 1 collagen gel and injected into the scaffold with a 22 gauge flat needle. The sample was photographed fluorescently using a $490 \mathrm{~nm}$ excitation filter and a $520 \mathrm{~nm}$ emission filter.

\subsection{Histology}

At 1, 7, 14 and 21 days, samples were stained and photographed. The tracheal rings were fixed in 10\% formalin. The specimens were embedded in paraffin and serial $5 \mu \mathrm{m}$ sections were cut in a coronal plane, mounted on glass slides, and stained with hematoxylin \& eosin and Safranin O/Fast Green. The samples were examined with a bright field microscope and digital images were captured.

\subsection{Biomechanics}

Samples were tested on day 0 and day 21 to see effects of cells and cellular activity on the scaffolds via uncon- 
fined compression under stress relaxation on a material test frame (Instron 5566) equipped with a $10 \mathrm{~N}$ load cell. Multiple step deformations were applied (5\%, 10\%, 15\% strain) and samples were allowed to equilibrate. Equilibrium stress-strain curves were analyzed to determine the Young's modulus.

\subsection{Statistical Analysis}

Proliferation data was examined by a two-tailed T-Test comparing the growth rates control chondrocytes versus chondrocytes in the 3D printed scaffold. Biomechanical group data was examined by one-way ANOVA. T-Test and ANOVA were performed using Statplus software (Analyst Soft) $(\alpha=0.05)$.

\section{Results}

Our results demonstrate the feasibility of 3D printing a CAD design of a tracheal ring that closely approximates normal tissue. The cell proliferation assay demonstrated that cells seeded in the scaffold had an average initial viability of $87.5 \%$ and cell number increased over 7 days to approximately twice the initial concentration (Figure 2). The cells grown in the scaffold demonstrated a similar proliferation trend compared to control cells (T-test comparing the proliferation rates; $\mathrm{p}=0.42$ ).

Biomechanical testing revealed that the cell-seeded scaffolds (group 3) retained their physical properties throughout the study when compared to non-treated controls (group 1 and 2). Cell seeding didn't alter the mechanical properties of the scaffolds during the study period. Specifically, no significant differences in the stiffness (Youngs Modulus) were identified over a 21-day period $(\mathrm{p}=0.99)$ (Table 1).

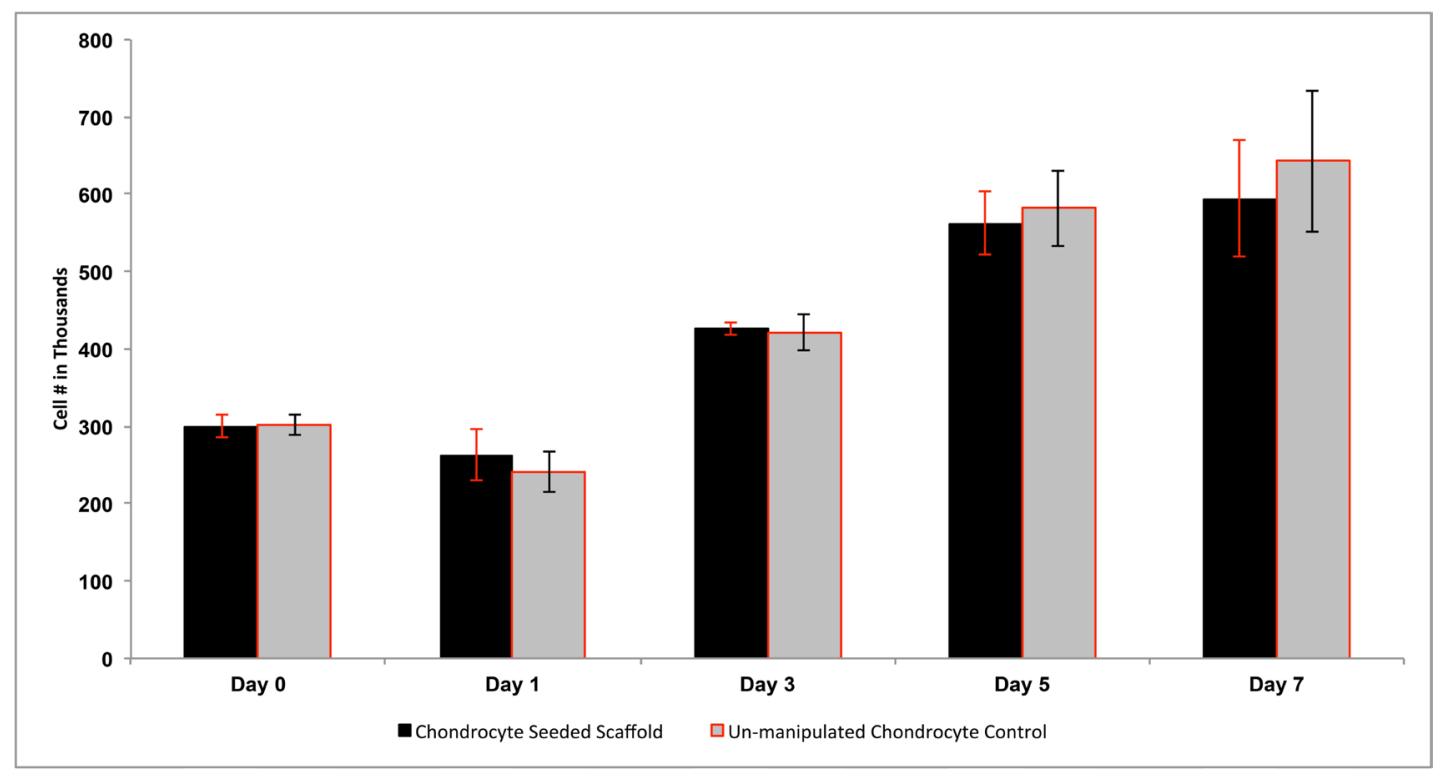

Figure 2. Cell proliferation assay over a 7-day period, demonstrating similar proliferation rates for cells seeded in the scaffold versus controls (T-test comparing the proliferation rates; $\mathrm{p}=0.42$ ).

Table 1. Biomechanical analysis of empty scaffold (group 1), collagen only scaffold (group 2), and chondrocyte seeded (group 3) at 0 and 21 days. Variance = standard deviation.

\begin{tabular}{|c|c|c|c|c|c|}
\hline \multicolumn{2}{|l|}{ Summary of Youngs Modulus (kPa) } & \multicolumn{2}{|c|}{0 Days } & \multicolumn{2}{|c|}{21 Days } \\
\hline Groups & Sample Size & Mean & Variance & Mean & Variance \\
\hline 1) Empty Control & 5 & 0.774 & 0.009 & 0.762 & 0.010 \\
\hline 2) Collagen Only & 5 & 0.774 & 0.010 & 0.768 & 0.009 \\
\hline 3) Cell Seeded & 5 & 0.763 & 0.011 & 0.772 & 0.006 \\
\hline \multicolumn{6}{|l|}{ ANOVA } \\
\hline Source of Variation & & & & & \\
\hline Between Groups: & & & & & \\
\hline
\end{tabular}


Histology of the cell seeded scaffold 1 day after incubation showed equal distribution of cells throughout the graft (Figure 3). Upon visual inspection, the cells appeared to retain their characteristic round shape and equal distribution throughout the 21-day study period. Numerous islands of Safranin-O positive staining were seen within the scaffold, demonstrating the presence of aggrecan components. The quantity of Safranin-O positive staining increased over the 21-day study period indicating that the chondrocytes retained their biological properties (Figure 4).

\section{Discussion}

Patients with long segment tracheal stenosis or malacia or patients who require revision tracheal resection represent clinical challenges for the airway surgeon. Allogeneic tracheal transplantation can provide an excellent functional result, but requires lifelong immunosuppression and is dependent on the supply of donor tracheas [9]. To

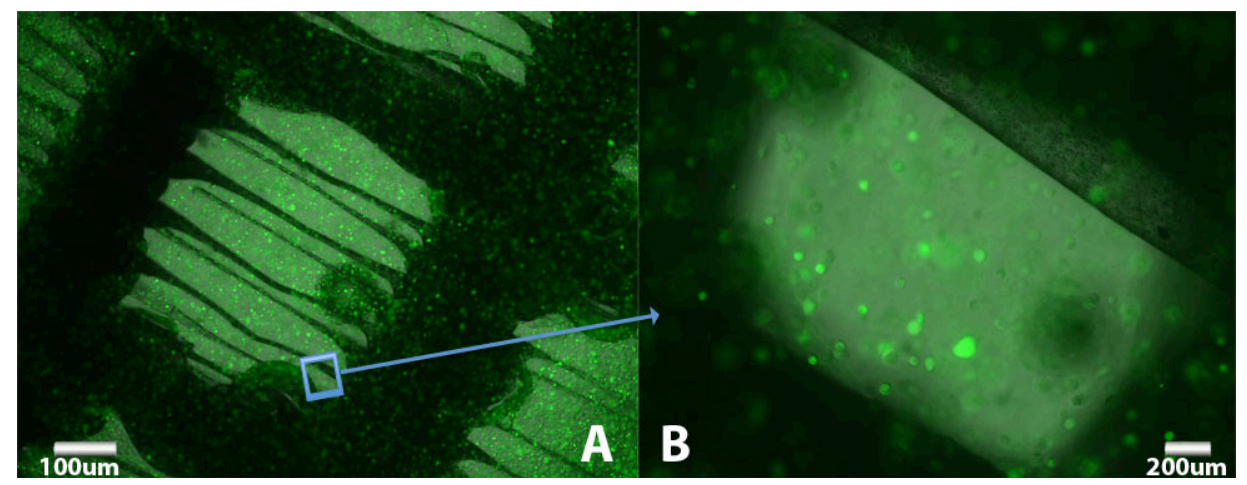

Figure 3. Fluorescently labeled, chondrocytes seeded (shown as green) scaffold at 1 day (A) 10× (B) $40 \times$ of the base if the ring.

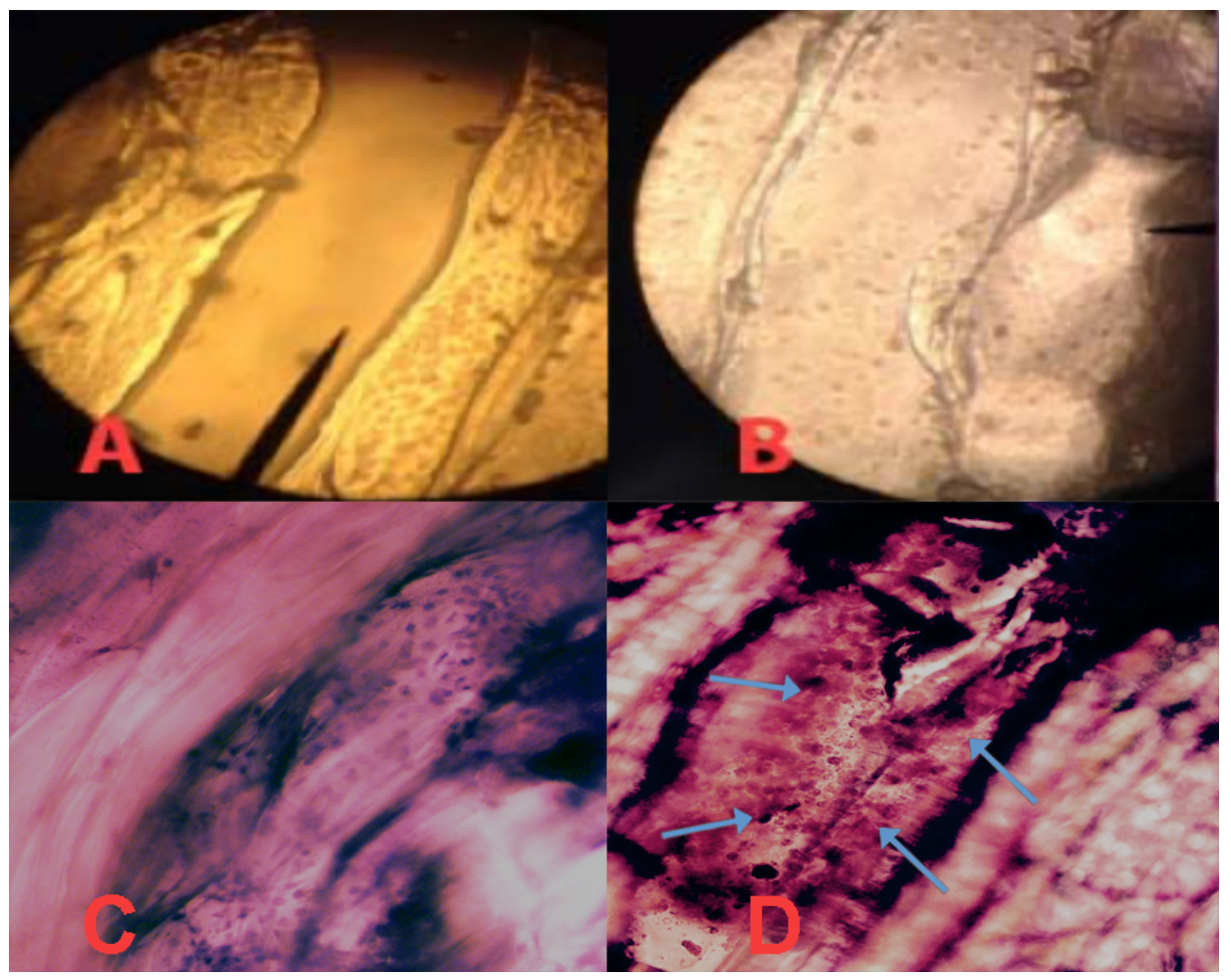

Figure 4. Microscopy of the base of the ring with chondrocytes (10×) (A). 1 day (B). 21 days (C). Safranin-O/Fast Green staining at 7 days (D). Safranin-O/Fast Green staining at 21 days. Safranin-0 positive staining indicates (arrows) the presence of proteoglycan clusters within the scaffold. 
address the issue of immunosuppression, some surgeons have proposed transplanting devitalized tracheal allografts [10]. These grafts are treated by washing away all donor cellular material, rendering them immunologically inert, and mitigating the need for the immune suppression normally required for transplantation [10]. The grafts are then seeded with stem cells from the recipient to produce true biological replacement prosthesis. The allograft itself serves merely as a biological scaffold on which to seed recipient stem cells prior to transplantation [5]. While this is a promising development, it requires extensive time and cost, and can result in biomechanical degradation requiring stenting [9] [11].

Another promising development is the emergence of 3D printing technology in which digital designs can be virtually modeled and printed into the physical world with precision. If this technology can be adapted into the realm of tracheal reconstruction, 3D printing can allow for individualized constructs with ideal biomechanical properties and physiologic function. Indeed, surgeons have already attempted to address airway malacia by means of external airway splinting using biodegradable 3D printed scaffolds without chondrocytes [3] [12]. Animal models exploring the biology underlying this approach have yielded improved respiration, but premature splint degradation or improper fit can cause a recurrence of tracheomalacia [13]. This failure may be attributed to the lack of cartilaginous in growth to support the trachea as the splint degrades.

The combination of living cells, whose proliferation rates are comparable to native cells [14] [15], and a 3D modeled patient specific graft may address some of these failures. In this study we demonstrate the ability to fabricate a customized 3D printed biodegradable PLA scaffold containing proliferating chondrocytes. Our data indicate that the cells within the scaffold are viable and proliferate and retain their biological function by synthesizing extracellular matrix. As well, when comparing Young's modulus of native cartilage tissue to the produced scaffold, has higher values of $0.77 \mathrm{kPa}$ compared to an average of $0.26 \mathrm{kPa}$ of native cartilage [16]. This proof of concept study represents a first step towards producing a 3D printed and tissue engineered long segment tracheal replacement graft for airway surgery.

Other groups have recently studied tracheal replacement grafts with some level of success [15] [17]. However, all these approaches have potential drawbacks, including a complex scaffold fabrication process which prohibits widespread use, use of stem cells with unknown long-term phenotype, and use of growth factors in the incubation phase with unknown neoplastic potential [15] [17]. Our approach involves the creation of a 3D printed CAD design and optimizes that design using commercially available software. The design can then be 3D printed using a readily available device. PLA is chosen to fabricate the scaffold, as it is FDA approved for various uses ranging from bone screws to wrinkle reducing injections. We are then able to seed the scaffold with mature chondrocytes, which survive and retain their biological function, thus avoiding the risks associated with stem cells and/or growth factors.

Although the complex and specialized technology is extremely valuable in solving many biomedical problems, the cost can be prohibitive for many institutions. With our model, we have shown that widely available technology, combined with innovative optimization, can make these solutions accessible to almost any health care facility. In addition to accessibility, our proof of concept model of tissue engineered cartilaginous grafts can be utilized for various medical purposes. 3D printed tissue engineered constructs using a patient's own cells allow for many new implantable applications far beyond the trachea. Future directions include exploring the use of other polymers with varying degradation times and expanding the application of this technology to address other unmet challenges in otolaryngology, such as auricular reconstruction, facial contouring, and nasal reconstruction.

\section{References}

[1] Berg, M., Ejnell, H., Kovács, A, et al. (2014) Replacement of a Tracheal Stenosis with a Tissue-Engineered Human Trachea Using Autologous Stem Cells: A Case Report. Tissue Engineering Part A, 20, 389-397. http://dx.doi.org/10.1089/ten.tea.2012.0514

[2] Jungebluth, P., Bader, A., Baiguera, S., et al. (2012) The Concept of in Vivo Airway Tissue Engineering. Biomaterials, 33, 4319-4326. http://dx.doi.org/10.1016/j.biomaterials.2012.03.016

[3] Ernst, A., Majid, A., Feller-kopman, D., et al. (2007) Airway Stabilization with Silicone Stents for Treating Adult Tracheobronchomalacia: A Prospective Observational Study. Chest, 132, 609-616. http://dx.doi.org/10.1378/chest.06-2708

[4] Xu, Y., Fan, F., Kang, N., et al. (2015) Tissue Engineering of Human Nasal Alar Cartilage Precisely by Using Three-Dimensional Printing. Plastic and Reconstructive Surgery, 135, 451-458. 
http://dx.doi.org/10.1097/PRS.0000000000000856

[5] Kojima, K., Bonassar, L.J., Ignotz, R.A., Syed, K., Cortiella, J. and Vacanti, C.A. (2003) Comparison of Tracheal and Nasal Chondrocytes for Tissue Engineering of the Trachea. The Annals of Thoracic Surgery, 76, 1884-1888. http://dx.doi.org/10.1016/S0003-4975(03)01193-7

[6] Nabzdyk, C., Pradhan, L., Molina, J., Perin, E., Paniagua, D. and Rosenstrauch, D. (2009) Review: Auricular Chondrocytes-From Benchwork to Clinical Applications. In Vivo, 23, 369-380.

[7] Lavrentieva, A., Hatlapatka, T., Neumann, A., Weyand, B. and Kasper, C. (2013) Potential for Osteogenic and Chondrogenic Differentiation of MSC. Advances in Biochemical Engineering, 129, 73-88. http://dx.doi.org/10.1007/10_2012_133

[8] Morrison, R.J., Hollister, S.J., Niedner, M.F., et al. (2015) Mitigation of Tracheobronchomalacia with 3D-Printed Personalized Medical Devices in Pediatric Patients. Science Translational Medicine, 7, 285-264. http://dx.doi.org/10.1126/scitranslmed.3010825

[9] Jungebluth, P., Alici, E., Baiguera, S., et al. (2011) Tracheobronchial Transplantation with a Stem-Cell-Seeded Bioartificial Nanocomposite: A Proof-of-Concept Study. The Lancet, 378, 1997-2004. http://dx.doi.org/10.1016/S0140-6736(11)61715-7

[10] Jungebluth, P. and Macchiarini, P. (2014) Airway Transplantation. Thoracic Surgery Clinics, 24, 97-106. http://dx.doi.org/10.1016/j.thorsurg.2013.09.005

[11] Gonfiotti, A., Jaus, M.O., Barale, D., et al. (2014) The First Tissue-Engineered Airway Transplantation: 5-Year Follow-Up Results. The Lancet, 383, 238-244. http://dx.doi.org/10.1016/S0140-6736(13)62033-4

[12] Crowley, C., Birchall, M. and Seifalian, A.M. (2014) Trachea Transplantation: From Laboratory to Patient. Journal of Tissue Engineering and Regenerative Medicine, 9, 357-367.

[13] Zopf, D.A., Hollister, S.J., Nelson, M.E., Ohye, R.G. and Green, G.E. (2013) Bioresorbable Airway Splint Created with a Three-Dimensional Printer. New England Journal of Medicine, 368, 2043-2045. http://dx.doi.org/10.1056/NEJMc1206319

[14] Masters, I.B. and Chang, A.B. (2005) Interventions for Primary (Intrinsic) Tracheomalacia and Bronchomalacia in Children. Cochrane Database of Systematic Reviews, 4, Article ID: CD005304.

[15] Chang, J.W., Park, S.A., Park, J.K., et al. (2014) Tissue-Engineered Tracheal Reconstruction Using Three-Dimensionally Printed Artificial Tracheal Graft: Preliminary Report. Artificial Organs, 38, E95-E105. http://dx.doi.org/10.1111/aor.12310

[16] Korhonen, R.K., Laasanen, M.S., Töyräs, J., Rieppo, J., Hirvonen, J., Helminen, H.J. and Jurvelin, J.S. (2002) Comparison of the Equilibrium Response of Articular Cartilage in Unconfined Compression, Confined Compression and Indentation. Journal of Biomechanics, 35, 903-909. http://dx.doi.org/10.1016/S0021-9290(02)00052-0

[17] Komura, M., Komura, H., Kanamori, Y., et al. (2008) An Animal Model Study for Tissue-Engineered Trachea Fabricated from a Biodegradable Scaffold Using Chondrocytes to Augment Repair of Tracheal Stenosis. Journal of Pediatric Surgery, 43, 2141-2146. http://dx.doi.org/10.1016/j.jpedsurg.2008.08.038 